\title{
Molecular Hanle effect in the Paschen-Back regime
}

\author{
A. I. Shapiro ${ }^{1}$, D. M. Fluri ${ }^{1}$, S. V. Berdyugina ${ }^{1,2}$, and J. O. Stenflo ${ }^{1,3}$ \\ 1 Institute of Astronomy, ETH Zurich, 8092 Zurich, Switzerland \\ e-mail: shapiro@astro.phys.ethz.ch \\ 2 Astronomy Division, PO Box 3000, 90014 University of Oulu, Finland \\ ${ }^{3}$ Faculty of Mathematics \& Science, University of Zurich, Germany
}

Received 13 July 2006 / Accepted 23 September 2006

ABSTRACT

\begin{abstract}
Context. The second solar spectrum resulting from coherent scattering is a main tool for diagnostics of turbulent magnetic fields on the Sun. Scattering on diatomic molecules plays an important role in forming this spectrum and even dominates in some spectral regions.

Aims. In a magnetic field electronic states of a molecule are often perturbed via the Paschen-Back effect. Sometimes this perturbation can completely change the spectrum, not only quantitatively, but even qualitatively. Here we calculate molecular scattering properties taking into account the Paschen-Back effect.

Methods. Starting with the Hund's case (a) wave functions as a basis we obtain with the perturbation theory wave functions of the intermediate Hund's case (a-b) in a magnetic field. Using new, perturbed values of the Landé factors and transition amplitudes we calculate the Mueller matrix for coherent scattering at diatomic molecules in the intermediate Hund's case (a-b) and look for the effects that can be caused by the Paschen-Back effect.

Results. We have found a considerable deviation from the Zeeman regime and discuss here the quantitative and qualitative effects on observed polarization signals for the $\mathrm{CN} B^{2} \Sigma-X^{2} \Sigma$ and $\mathrm{MgH} B^{\prime 2} \Sigma-X^{2} \Sigma$ systems as examples.
\end{abstract}

Key words. line: formation - magnetic fields - molecular processes - polarization - radiative transfer - scattering

\section{Introduction}

The properties of the coherent scattering process are modified in a magnetic field via the Hanle effect, which provides us with a very sensitive tool for studying the distribution of weak magnetic fields on the Sun. The Hanle effect is most sensitive when the magnetic splitting is comparable to the natural line width. For most atomic transitions this implies weak magnetic fields, so that the level splitting can be calculated in the Zeeman effect regime (ZR). For many molecular transitions even weak magnetic fields can significantly perturb the internal molecular structure and change wave functions of molecular levels. Therefore the level splitting and amplitudes of the transitions should be calculated taking into account the Paschen-Back effect (PBE) regime (van Vleck 1926; Hill 1929; Schadee 1978; Berdyugina et al. 2005; Asensio Ramos \& Trujillo Bueno 2006).

Previously the Hanle effect in the Paschen-Back regime (PBR) was treated only for atomic transitions by Bommier (1980) and Landolfi \& Landi Degl'Innocenti (1985), who studied the Hanle effect on level-crossings for the helium $D_{3}$ and sodium D lines of solar prominences.

The recent first analysis of molecular scattering polarization (Berdyugina et al. 2002; Faurobert \& Arnaud 2003; Landi Degl'Innocenti 2003; Trujillo Bueno 2003; Asensio Ramos \& Trujillo Bueno 2005; Bommier et al. 2006) and the first detection of the Hanle effect in molecular lines on the Sun (Berdyugina \& Fluri 2004) revealed the clear advantages of molecular lines for studying turbulent magnetic fields by means of the differential Hanle effect, and thus the necessity to expand the theory of the Hanle effect taking into account the $\mathrm{PBE}$ for the molecular case.
Here, for the first time, we couple the PBE with the Hanle effect in molecular lines. In Sect. 2 we shortly introduce the Paschen-Back effect, before we discuss its general influence on linear polarization. In Sect. 3 we give an overview of the Hanle effect theory and present our method of calculation, emphasizing distinctions between the Zeeman regime (ZR) and Paschen-Back regime (PBR) calculations. In Sect. 4 we discuss the PBE influence on scattering profiles. Preliminary results on single line calculations have been previously reported by Shapiro et al. (2006). Here we present more general calculations for multiplet transitions. Finally in Sect. 5 we discuss net polarization properties of scattered radiation and the Hanle effect in the saturated regime.

We illustrate all our general conclusions with example calculations for the $\mathrm{CN} B^{2} \Sigma-X^{2} \Sigma$ and $\mathrm{MgH} B^{\prime 2} \Sigma-X^{2} \Sigma$ systems, which are strongly influenced by the PBE even for weak fields.

\section{Paschen-Back effect}

The PBE results from the perturbation between different molecular or atomic states in the presence of a magnetic field. These interactions change both positions and intensities of lines, which can completely alter the appearance of the spectrum both quantitatively and qualitatively.

There are different possibilities for the PBE in molecules. It can occur, for example, on the fine structure, rotational structure or between different electronic states. Usually the effect between different electronic states becomes important only for quite strong magnetic fields. In our calculations we take into account only the first two, i.e. on the fine and rotational structures. The relative importance of these effects and the sequence of their appearance with increasing magnetic field strength depends on 


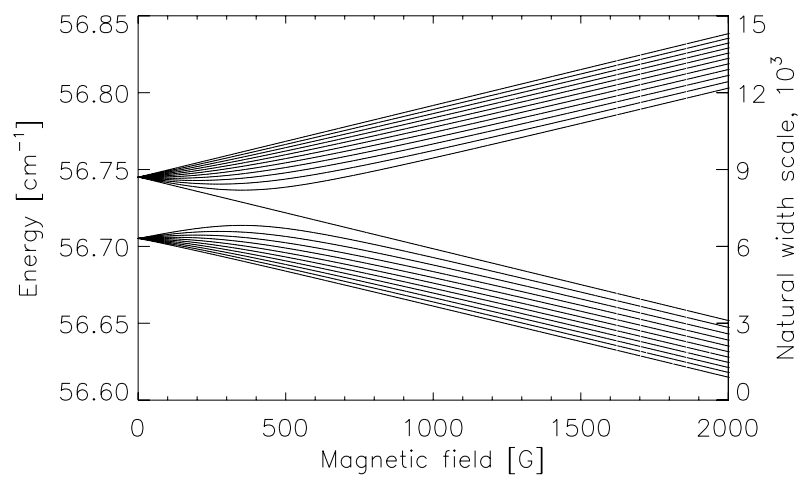

Fig. 1. The Paschen-Back effect in the doublet level with $N=5$ for the $B^{2} \Sigma v=0$ state of $\mathrm{CN}$.

molecular constants. In the limiting Hund's case (a) (strong spinorbital coupling), when the rotational splitting is smaller than the fine structure splitting, the PBE on the rotational structure first occurs. On the contrary, in the limiting Hund's case (b) (weak spin-orbital coupling), the PBE on the fine structure first occurs. Our calculations of the PBE are based on works of Kovács (1969), Schadee (1978), Berdyugina et al. (2005) and can be applied to the intermediate Hund's case (a-b).

In this paper we provide example calculations for $\Sigma-\Sigma$ transitions, which are described by Hund's case (b). Because of the zero orbital momentum of the upper and lower states there is no PBE on the rotational structure, but there is a quite strong effect on the fine structure of both states due to small spin-rotational constants. In this case we therefore calculate the PBE only on the fine structure.

The level splitting as a function of the magnetic field strength is shown in Fig. 1 for the doublet with $N=5$ of the $B^{2} \Sigma v=0$ state of $\mathrm{CN}$ (upper doublet component with $J=5.5$, lower with $J=4.5$ ). With the increasing magnetic field strength the electron spin gradually decouples from the rotational momentum, resulting in modification of the Landé factors. The PBE becomes apparent in the splitting pattern above about $100 \mathrm{G}$, while towards $2 \mathrm{kG}$ the doublet approaches the complete PBE regime, when the spin is fully decoupled from the rotation. In this case the sublevels separate in two groups, according to the spin projection on the magnetic field direction: $\mu_{\mathrm{S}}=+1 / 2$ and $\mu_{\mathrm{S}}=-1 / 2$ (Herzberg 1950; Berdyugina et al. 2005). Note also that the levels with magnetic numbers $\mu= \pm J$ in the upper doublet state split linearly as in the ZR (because for them there are no levels to interact with in the fine structure).

The PBE also changes the amplitudes of transitions. The dependence of Hönl-London factors on the magnetic field strength for the $\mathrm{CN}(0,0)$ band transitions is given in the upper part of Fig. 2 for main (left panel) and satellite (right panel) rotational branches. Because of the mixing of states with different $J$-values the $\mathrm{O}$ and $\mathrm{S}$ branches appear, which are forbidden in the zero-field case. They reach maximum strengths in the intermediate PBR, and decrease down to zero when approaching the complete PBR. Note, that in this case (the PBE only on the fine structure) the sum of the Hönl-London factors for all lines of the multiplet is constant and the PBE appears only in the strength redistribution between different multiplet components.

The mixing of levels implies that $J$-values lose their meaning and become "bad" quantum numbers. Therefore the definition of rotational branches strictly holds only in the ZR.

Since magnetic sublevels are grouped according to the spin magnetic number $\mu_{\mathrm{S}}$ for large magnetic fields (Fig. 1), we recalculate Hönl-London factors summing up transitions according to the value of $\mu_{\mathrm{S}}$. Then, four groups of transitions with $\Delta \mu_{\mathrm{S}}=0, \pm 1$ can in principle be distinguished. They are shown in the lower panels of Fig. 2. In the complete PBE the transitions with $\Delta \mu_{\mathrm{S}}= \pm 1$ are forbidden (Herzberg 1950), so their strengths approach zero as the field increases.

In the cases close to the complete PBE regime these recalculated Hönl-London factors are more representative, because transitions between sublevels will group according to their $\Delta \mu_{\mathrm{S}}$ value as well.

\section{Scattering processes}

\subsection{General theory of the Hanle effect}

To identify the place where the alterations due to the PBE do come from we give here a short overview of coherent scattering theory, following the description according to Landi Degl'Innocenti \& Landi Degl'Innocenti (1988), Stenflo (1994, 1997, 1998) and Landi Degl'Innocenti \& Landolfi (2004).

A radiation field at a fix point of space can be characterized by the Stokes vector, which generally changes in a scattering process. To describe the relation between the incident and scattered Stokes vectors the Mueller matrix $\mathbf{M}$ or coherency matrix $\mathbf{W}$ (transformed Mueller matrix: $\mathbf{W}=\mathbf{T}^{-\mathbf{1}} \mathbf{M T}$, where $\mathbf{T}$ is the transformation matrix) are used.

In the zero field case the Mueller matrix for scattering of one component of a multiplet can be factorized into a scalar function depending only on frequency, and the phase matrix

$\mathbf{P}=W_{2} \mathbf{P}_{\mathbf{R}}+\left(1-W_{2}\right) \mathbf{E}_{\mathbf{1 1}}$,

which describes all the polarization properties. Here $\mathbf{P}_{\mathbf{R}}$ is the Rayleigh phase matrix, while the $\mathbf{E}_{\mathbf{1 1}}$ matrix (first diagonal element is one, the rest zero) represents unpolarized, isotropic scattering. Therefore the coefficient $W_{2}$, the so-called effective scattering polarizability, branches polarized and unpolarized scattering. It depends only on transition strengths between magnetic sublevels. There is a well-known expression for $W_{2}$ (Stenflo 1994):

$W_{2}=\frac{\sum f_{\mu_{\mathrm{u}} \mu_{\mathrm{f}}}^{J_{\mathrm{u}} J_{\mathrm{f}}} f_{\mu_{\mathrm{u}} \mu_{\mathrm{i}}}^{J_{\mathrm{u}} J_{\mathrm{i}}}-\sum f_{\mu_{\mathrm{u}} \mu_{\mathrm{f}}}^{J_{\mathrm{u}} J_{\mathrm{f}}} f_{\mu_{\mathrm{u}} \mu_{\mathrm{i}}-1}^{J_{\mathrm{u}} J_{\mathrm{i}}}}{\sum f_{\mu_{\mathrm{u}} \mu_{\mathrm{f}}}^{J_{\mathrm{f}} J_{\mathrm{f}}} f_{\mu_{\mathrm{u}} \mu_{\mathrm{i}}}^{J_{\mathrm{u}} J_{\mathrm{i}}}+2 \sum f_{\mu_{\mathrm{u}} \mu_{\mathrm{f}}}^{J_{\mathrm{u}} J_{\mathrm{f}}} f_{\mu_{\mathrm{u}} \mu_{\mathrm{i}}-1}^{J_{\mathrm{u}} J_{\mathrm{i}}}}$

where all the summations are done under the condition $\mu_{\mathrm{u}}=\mu_{\mathrm{f}}=$ $\mu_{\mathrm{i}}$, and $f_{\mu_{a} \mu_{b}}^{J_{a} J_{b}}$ are the strengths of transitions between $\left(J_{a}, \mu_{a}\right)$ and $\left(J_{b}, \mu_{b}\right)$ substates. Here $\mathrm{i}, \mathrm{u}$ and $\mathrm{f}$ are initial, intermediate and final substates, respectively.

In a magnetic field the phase matrix $\mathbf{P}$ is modified due to the Hanle effect. To take this into account and to perform the calculation for the whole multiplet (which is especially important in case of the PBR) it is necessary to account accurately for all the scattering levels and interferences between them.

The coherency matrix of a scattering process from the set of initial substates $i$ via the intermediate substates $u$ to the final substates $\mathrm{f}$ is given by

$\mathbf{W}=\sum_{J_{\mathrm{i}}, \mu_{\mathrm{i}}} \sum_{J_{\mathrm{f}}, \mu_{\mathrm{f}}} \boldsymbol{w}\left(J_{\mathrm{i}}, \mu_{\mathrm{i}} ; J_{\mathrm{f}}, \mu_{\mathrm{f}}\right) \otimes \boldsymbol{w}\left(J_{\mathrm{i}}, \mu_{\mathrm{i}} ; J_{\mathrm{f}}, \mu_{\mathrm{f}}\right)^{*}$,

where the incoherent summation is done over all total momentum and magnetic quantum numbers of the initial and final states $J_{\mathrm{i}, \mathrm{f}}, \mu_{\mathrm{i}, \mathrm{f}}$. Here $\boldsymbol{w}\left(J_{\mathrm{i}}, \mu_{\mathrm{i}}, J_{\mathrm{f}}, \mu_{\mathrm{f}}\right)$ is the Jones matrix for scattering with the fixed quantum numbers of the initial and 

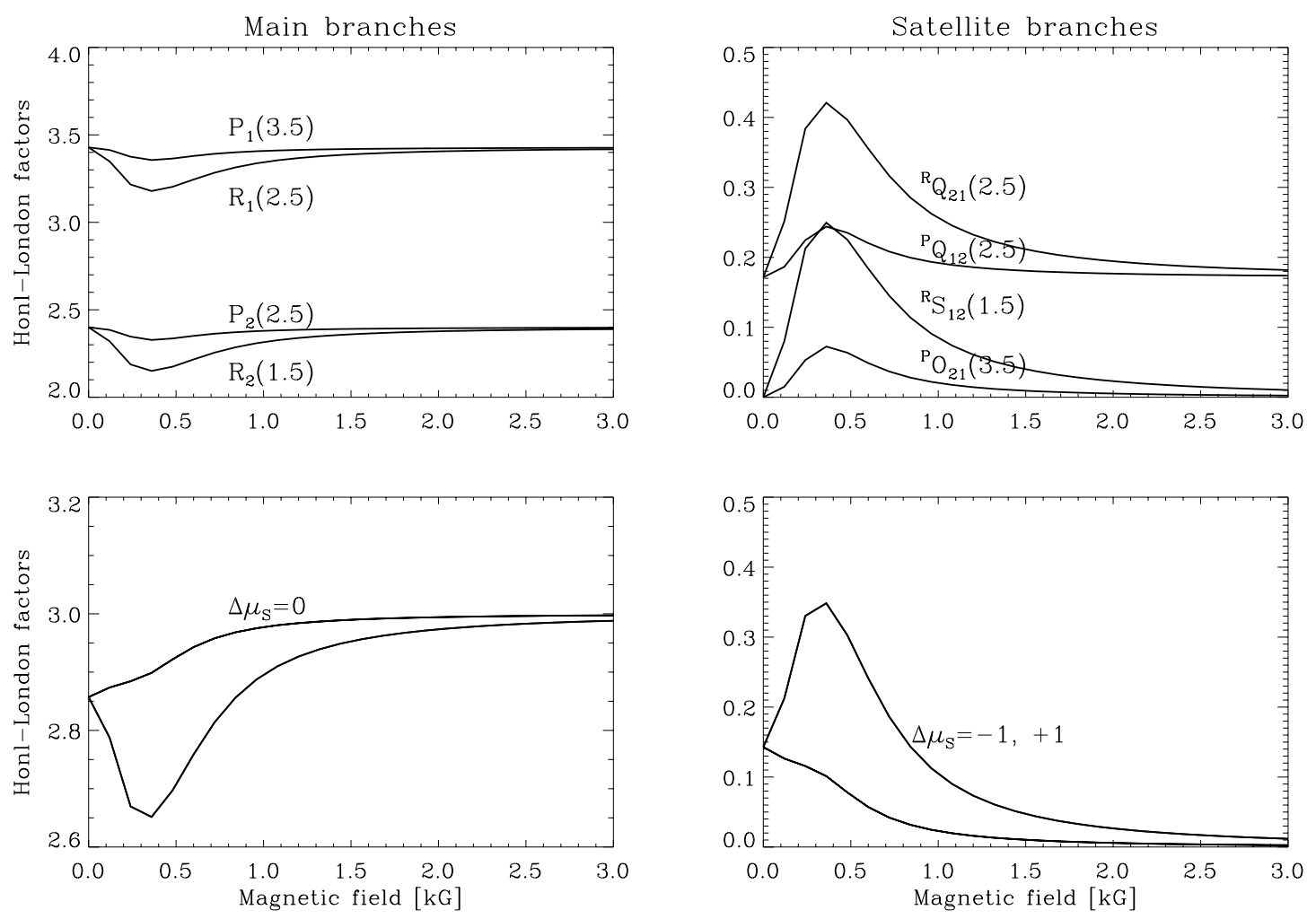

Fig. 2. Dependence of the Hönl-London factors on magnetic field strength for the $\mathrm{CN}$ violet system. Upper panels: calculations for transitions corresponding to the different rotational branches, which are strictly defined only for weak magnetic fields. Lower panels: the grouping is done for transitions between states with fixed spin projection on the magnetic field direction, a definition which is representative of the physics in the complete PBR.

final states. Its elements are given by the Kramers-Heisenberg formula

$w_{\alpha \beta} \sim \sum_{J_{\mathrm{u}}, \mu_{\mathrm{u}}} \frac{\left\langle J_{\mathrm{f}}, \mu_{\mathrm{f}}\left|\hat{\boldsymbol{r}} \cdot \boldsymbol{e}_{\alpha}\right| J_{\mathrm{u}}, \mu_{\mathrm{u}}\right\rangle\left\langle J_{\mathrm{u}}, \mu_{\mathrm{u}}\left|\hat{\boldsymbol{r}} \cdot \boldsymbol{e}_{\beta}\right| J_{\mathrm{i}}, \mu_{\mathrm{i}}\right\rangle}{\omega_{\mathrm{uf}}-\omega-\mathrm{i} \gamma / 2}$,

where the coherent summation is done over all total momentum and magnetic quantum numbers $J_{\mathrm{u}}, \mu_{\mathrm{u}}$ of the intermediate substate $\mathrm{u}$. In this formula $\boldsymbol{e}_{\alpha}$ and $\boldsymbol{e}_{\beta}(\alpha, \beta=1,2)$ are the unit polarization vectors of emission and absorption, respectively, $\omega$ is the frequency of the scattered radiation, $\omega_{\mu_{\mathrm{u}} \mu_{\mathrm{f}}}$ is the frequency corresponding to the energy difference between the upper and lower states, and $\gamma$ is the damping constant.

The Jones matrix elements in Eq. (4) can also be written in an explicit form:

$w_{\alpha \beta} \sim \sum_{J_{\mathrm{u}}, \mu_{\mathrm{u}}}(-1)^{q_{\mathrm{f}}-q_{\mathrm{i}}} Q_{J_{\mathrm{i}}, \mu_{\mathrm{i}}}^{J_{\mathrm{u}}, \mu_{\mathrm{u}}} Q_{J_{\mathrm{f}}, \mu_{\mathrm{f}}}^{J_{\mathrm{u}}, \mu_{\mathrm{u}}} \varepsilon_{q_{\mathrm{f}}}^{\alpha *} \varepsilon_{q_{\mathrm{i}}}^{\beta} \phi\left(v-v_{J_{\mathrm{f}}, \mu_{\mathrm{f}}}^{J_{\mathrm{u}}, \mu_{\mathrm{u}}}\right)$.

Here, $q_{\mathrm{f}}=\mu_{\mathrm{f}}-\mu_{\mathrm{u}}, q_{\mathrm{i}}=\mu_{\mathrm{i}}-\mu_{\mathrm{u}}, Q_{a}^{b}$ is the amplitude of the transition between the lower state with quantum numbers $a$ and the upper state with quantum numbers $b, \phi(v)$ is the normalized zero-centered Lorentz profile, and $\varepsilon_{q_{\mathrm{f}}, q_{\mathrm{i}}}^{\alpha, \beta}=\boldsymbol{e}_{q_{\mathrm{f}}, q_{\mathrm{i}}} \cdot \boldsymbol{e}_{\alpha, \beta}$ are geometrical factors, where $\boldsymbol{e}_{q}$ are complex spherical vectors. It is important that all transition amplitudes $Q$ in Eq. (5) have the correct sign (cf. Schadee 1978; Stenflo 1994).

The basic feature of Eqs. (4) and (5) is the coherent summation over all quantum numbers $J_{\mathrm{u}}, \mu_{\mathrm{u}}$ of the intermediate states $\mathrm{u}$, since it is the source of the interference terms. In contrast, Eq. (3) only describes blending of lines, arising from non-interfering transitions with fixed initial and final quantum numbers.
Substituting Eq. (5) in Eq. (3) we obtain

$$
\mathbf{W}=\sum_{\mathrm{i}, \mathrm{f}} \sum_{\mathrm{u}, \mathrm{u}^{\prime}} A_{\mathrm{J}_{\mathrm{i}} \mathrm{J}_{\mathrm{f}} \mathrm{J}_{\mathrm{u}} J_{\mathrm{u}^{\prime}}}^{\mu_{\mathrm{i}} \mu_{\mathrm{u}} \mu_{\mathrm{u}} \mu^{\prime}} \Phi\left(v-v_{\mathrm{f}}^{\mathrm{u}}, v-v_{\mathrm{f}}^{\mathrm{u}^{\prime}}\right) \boldsymbol{\Gamma}\left(q_{\mathrm{i}}, q_{\mathrm{f}}, q_{\mathrm{i}}{ }^{\prime}, q_{\mathrm{f}}{ }^{\prime}\right) .
$$

Here $A$ is a product of four transition amplitudes (with signs). It depends only on the quantum numbers of the initial, final and two intermediate states. The function $\Phi\left(v-v_{\mathrm{f}}^{\mathrm{u}}, v-\right.$ $\left.v_{\mathrm{f}}^{\mathrm{u}^{\prime}}\right)=\phi\left(v-v_{\mathrm{u}}\right) \phi\left(v-v_{\mathrm{u}^{\prime}}\right)$ specifies the dependence on frequency of the scattered photon. The $4 \times 4$ matrix $\Gamma\left(q_{\mathrm{i}}, q_{\mathrm{f}}, q_{\mathrm{i}}{ }^{\prime}, q_{\mathrm{f}}{ }^{\prime}\right)=$ $\left(\varepsilon_{q_{\mathrm{f}}}^{\alpha *} \varepsilon_{q_{\mathrm{i}}}^{\beta}\right) \otimes\left(\varepsilon_{q_{\mathrm{f}}}^{\alpha *} \varepsilon_{q_{\mathrm{i}}{ }^{\prime}}^{\beta}\right)^{*}$ is defined only by the scattering geometry (directions of the incident and scattered radiation, and of the magnetic field and polarizations vectors), $q_{\mathrm{i}, \mathrm{f}}, q_{\mathrm{i}, \mathrm{f}}{ }^{\prime}$ correspond to the interfering upper substates $u$ and $u^{\prime}$ substates.

According to the selection rules, many terms in Eq. (6) are zero. For example, if we fix the initial magnetic quantum number $\mu_{\mathrm{i}}$, only five different values for $\mu_{\mathrm{f}}$ are possible: $\mu_{\mathrm{f}}=\mu_{\mathrm{i}}$ (in this case there are three interfering magnetic substates with $\mu_{\mathrm{u}}=\mu_{\mathrm{i}}-1, \mu_{\mathrm{i}}, \mu_{\mathrm{i}}+1$ for each component of the fine structure); $\mu_{\mathrm{f}}=\mu_{\mathrm{i}}+1$ (interfering substates with $\mu_{\mathrm{u}}=\mu_{\mathrm{i}}, \mu_{\mathrm{i}}+1$ ); $\mu_{\mathrm{f}}=\mu_{\mathrm{i}}-1$ (interfering substates with $\left.\mu_{\mathrm{u}}=\mu_{\mathrm{i}}-1, \mu_{\mathrm{i}}\right) ; \mu_{\mathrm{f}}=\mu_{\mathrm{i}} \pm 2$ (no interference between excited substates). Altogether there are 14 different sets of $q_{\mathrm{i}, \mathrm{f}}, q_{\mathrm{i}, \mathrm{f}^{\prime}}$ values, and each of them defines one matrix $\Gamma$.

The representation of the coherency matrix in the form of Eq. (6) allows us to separate the dependences on geometry, frequency and transition amplitudes in the calculations. For example, we need to calculate all frequency-dependent coefficients $A$ only once, which greatly saves computing time, especially in the PBR, where transition amplitude calculations are the most timeconsuming. This representation is also useful for averaging the results over some distribution of magnetic field directions (for 
example, for turbulent fields). In this case we can vary the directions of the incident and scattered radiation while leaving the magnetic field direction unchanged in the chosen coordinate system. This is equivalent to changing the magnetic field direction, but it avoids redefinitions of the magnetic quantum numbers. Since all the geometric information is contained in the matrices $\boldsymbol{\Gamma}$, only these $144 \times 4$ matrices are to be averaged. For a fixed field direction distribution all matrix coefficients need to be calculated only once and can then be applied to calculations for arbitrary magnetic field strengths and quantum numbers. This again saves the computing time because precise averaging is quite time consuming.

The coherency matrix $\mathbf{W}$ in Eq. (6) is obtained assuming a Lorentz line profile. In the general case we have to convolve $\mathbf{W}$ with a Doppler profile. For this purpose the frequency-dependent function $\Phi$ is usually transformed to the following form (Stenflo 1994):

$$
\begin{aligned}
\Phi\left(v-v_{\mathrm{f}}^{\mathrm{u}}, v-v_{\mathrm{f}}^{\mathrm{u}^{\prime}}\right)= & C \cos \alpha_{q_{\mathrm{f}, \mathrm{f}^{\prime}}} \\
& \times \mathrm{e}^{\mathrm{i} \alpha_{q_{\mathrm{f}, \mathrm{f}^{\prime}}}\left(\phi\left(v-v_{\mathrm{u}}\right)+\phi\left(v-v_{\mathrm{u}^{\prime}}\right)\right) .}
\end{aligned}
$$

Here, $C$ is a normalization constant, while the Hanle angle $\alpha_{q_{\mathrm{f}, \mathrm{f}^{\prime}}}$ is defined by

$\tan \alpha_{q_{\mathrm{f}, \mathrm{f}^{\prime}}}=\frac{v_{\mathrm{f}}^{\mathrm{u}}-v_{\mathrm{f}}^{\mathrm{u}^{\prime}}}{\gamma_{N}+\gamma_{\mathrm{c}} / 2}$,

where $\gamma_{N}$ and $\gamma_{\mathrm{c}}$ are the radiative and collisional damping constants.

Convolving the function $\Phi\left(v-v_{\mathrm{f}}^{\mathrm{u}}, v-v_{\mathrm{f}}^{\mathrm{u}^{\prime}}\right)$ with the Doppler profile, we obtain from Eq. (7):

$$
\begin{aligned}
F\left(a, v-v_{\mathrm{f}}^{\mathrm{u}}, v-v_{\mathrm{f}}^{\mathrm{u}^{\prime}}\right) & \sim \cos \alpha_{q_{\mathrm{f}, \mathrm{f}^{\prime}}} \\
& \times \mathrm{e}^{\mathrm{i} \alpha_{q_{\mathrm{f}, \mathrm{f}^{\prime}}}}\left[\mathcal{H}\left(a, v-v_{\mathrm{f}}^{\mathrm{u}}\right)+\mathcal{H}^{*}\left(a, v-v_{\mathrm{f}}^{\mathrm{u} \prime}\right)\right]
\end{aligned}
$$

Here, $\mathcal{H}(a, \Delta v)=H(a, \Delta v)-2 \mathrm{i} F(a, \Delta v)$, with $H$ being the Voigt function and $F$ the dispersion function. Therefore, when we taking into account the Doppler broadening, we just have to replace the function $\Phi\left(v-v_{\mathrm{f}}^{\mathrm{u}}, v-v_{\mathrm{f}}^{\mathrm{u}^{\prime}}\right)$ with $F\left(a, v-v_{\mathrm{f}}^{\mathrm{u}}, v-v_{\mathrm{f}}^{\mathrm{u}^{\prime}}\right)$ in Eq. (6).

\subsection{Interferences}

The nature of the Hanle effect is the modification of the polarization due to interferences between the different possibilities of the scattering process in dependence of an external magnetic field. We can rewrite the summation in Eq. (6) in the following form:

$$
\begin{aligned}
\mathbf{W} & =\sum_{J_{\mathrm{i}, \mathrm{f}}, \mu_{\mathrm{i}, \mathrm{f}}}\left(\sum_{\substack{J_{\mathrm{u}}=J_{\mathrm{u}^{\prime}} \\
\mu_{\mathrm{u}}=\mu_{\mathrm{u}^{\prime}}}} A \cdot \Phi \cdot \boldsymbol{\Gamma}+\sum_{\substack{J_{\mathrm{u}}=J_{\mathrm{u}^{\prime}} \\
\mu_{\mathrm{u}} \neq \mu_{\mathrm{u}^{\prime}}}} A \cdot \Phi \cdot \boldsymbol{\Gamma}\right. \\
& \left.+\sum_{J_{\mathrm{u}} \neq J_{\mathrm{u}^{\prime}}} A \cdot \Phi \cdot \boldsymbol{\Gamma}\right),
\end{aligned}
$$

where for conciseness all variables of the $A, \Phi$ and $\boldsymbol{\Gamma}$ functions have been omitted. In this equation, the first sum in the brackets contains all diagonal terms, while the two other sums cover the interferences. For scattering processes through different intermediate states that are independent of each other, there will be only diagonal terms. The second sum in Eq. (10) contains the interference terms between different magnetic sublevels within one fine structure component. These terms have their maxima in the zero-field case, since without magnetic splitting all angles $\alpha_{q_{\mathrm{f}, \mathrm{f}^{\prime}}}$ are zero (see Eq. (8)). In a magnetic field non-zero angles (due to the magnetic splitting) reduce the interference terms, which in most cases leads to depolarization of the scattered radiation. Such modification of the polarization of the scattered radiation in a magnetic field is the essence of the Hanle effect. Finally the third sum in Eq. (10) contains interference terms between different components of the fine structure. Note that shifts of magnetic sublevels in a magnetic field also influence these terms. We see now that Eqs. (6) and (10) in their general form describe both the Hanle effect and the interference between different $J$-states.

If different fine or rotational structure components of the intermediate state are far from each other, we can fix the total angular momentum of the intermediate substates u in Eq. (4) and make the summation only over magnetic quantum numbers $\mu_{\mathrm{m}}$, neglecting the third sum in Eq. (10). This allows us to calculate the Hanle effect for scattering in each component of the multiplet separately. To account for the whole multiplet we can then sum incoherently the contributions from all multiplet components. On the other hand, if different intermediate $J$-states are close to each other, we have to coherently add the contributions from all the multiplet components and make the summation in Eq. (4) over all involved $J$-states.

\subsection{Alterations due to the PBE}

The general formulas presented above are valid for both the PBR and ZR. The PBE perturbs the Jones matrix by changing the amplitudes of interfering scattering processes $Q_{J_{\mathrm{i}}, \mu_{\mathrm{i}}}^{J_{\mathrm{u}}, \mu_{\mathrm{u}}} Q_{J_{\mathrm{f}}, \mu_{\mathrm{f}}}^{J_{\mathrm{u}}, \mu_{\mathrm{u}}}$ in Eq. (5) and contributions from them via the alterations of $v_{J_{\mathrm{f}}, \mu_{\mathrm{f}}}^{J_{\mathrm{u}}, \mu_{\mathrm{u}}}$ (in comparison with the Zeeman effect), while leaving all the geometric factors unchanged. Therefore we can distinguish two possible types of coherency matrix alterations due to the PBE. Firstly, the PBE modifies the absolute values of the terms in Eqs. (4) and (5). This effect alone can dramatically change the spectrum. Secondly, the different magnetic displacement in the PBE influences the phase of the terms in Eq. (5). This causes additional alterations of the interference terms in Eq. (10) and can even change their signs.

Doppler broadening should be done with care. In the ZR the numerator of the ratio in Eq. (8) is usually expressed as $\left(q_{\mathrm{f}}-\right.$ $\left.q_{\mathrm{f}}{ }^{\prime}\right) g_{\mathrm{u}} \omega_{L}$, where $g_{\mathrm{u}}$ is the Landé factor of the intermediate state and $\omega_{\mathrm{L}}$ is the Larmor frequency. In the PBR the magnetic sublevels are not equidistant, and Landé factors are dependent on the magnetic field strength and magnetic quantum numbers. Thus we need to use a more general expression, namely Eq. (8). Moreover, this equation can also be used for calculations with interferences between different components of the fine structure. Note that in the ZR both expressions are equivalent.

\section{Scattering line profiles in the PBR}

In the following we apply the theory developed above to investigate the influence of the PBE on scattering polarization profiles and illustrate our results with examples for the $\mathrm{CN}$ violet $\left(B^{2} \Sigma-X^{2} \Sigma\right)$ system and the $B^{\prime 2} \Sigma-X^{2} \Sigma$ system of $\mathrm{MgH}$. Therefore we will only deal with the PBE on the fine structure, which is the most interesting case for the molecular Hanle effect, but some of our conclusions are also valid for the PBE on the rotational and hyperfine structures. Here we give a more 
general calculation of a multiplet case compared to previous results by Shapiro et al. (2006). To emphasize the difference between the Hanle effect in the PBR and ZR we also carry out calculations neglecting the modifications of the transition amplitudes and Zeeman shifts due to the PBE. In the following this is called the ZR calculations.

For the PBE on the fine structure $N$ is a good quantum number, while $J$-states become mixed. Therefore, we calculate the scattering for the whole multiplet structure, making the summation over all $J$-states belonging to the $N$-multiplet in Eq. (6). We fix the lower $N_{1}$ and the upper $N_{\mathrm{u}}$ numbers and calculate the scattering profile from the $N_{\mathrm{l}} \rightarrow N_{\mathrm{u}} \rightarrow N_{\mathrm{l}}$ process, neglecting all transitions between levels with different $N$ numbers. This calculation contains eight scattering processes, corresponding to the zero-field definition of the rotational branches (as there are two ways of choosing $J$ in the zero-field case, for each of the states - initial, intermediate and final). For example, if $N_{\mathrm{u}}=N_{\mathrm{i}}+1$ we have to calculate the following transitions: $R_{1}-R_{1}, R_{1}-{ }^{R} S_{12}$, ${ }^{R} Q_{21}-{ }^{R} Q_{21},{ }^{R} Q_{21}-R_{2}, R_{2}-R_{2}, R_{2}-{ }^{R} Q_{21},{ }^{R} S_{12}-{ }^{R} S_{12},{ }^{R} S_{12}-R_{1}$. Three of these branches are forbidden in the ZR and appear only in the PBR.

\subsection{Alterations of the wing polarization}

The Hanle effect modifies the wing polarization in the PBR, which is not the case in the ZR. As we see from Eq. (4) the efficiency of the Hanle effect strongly depends on the magnetic displacement, which is implicitly contained in the $\omega_{\text {uf }}$ term, and on the transition amplitudes. Both these quantities are subject to the PBE. However, in the line wings $\omega-\omega_{\text {uf }}$ is much larger than the magnetic splitting of the line, which therefore becomes negligible. Nonetheless, due to the changed transition amplitudes the PBE influences the scattering polarization over the whole multiplet and produces modifications of the polarization in both the line cores and wings. In contrast, the Zeeman effect only leads to level shifts (without affecting transition amplitudes) and thus modifies the scattering polarization only in the line core, leaving the wing linear polarization unchanged for each component of the fine structure. Therefore, if we neglect the interference from nearby members of the same multiplet, the linear polarization in the wing of each multiplet component will in the ZR not depend on the magnetic field (both direction and strength) and can be calculated with Eqs. (1) and (2). In the PBR the situation is different.

The observable strengths of the components depend on magnetic field direction. The PBE breaks the balance between the $\sigma$ and $\pi$ components, whose polarization signals depend on the magnetic field direction. Therefore, the wing polarization depends on the field direction as well (in contrast if we in the ZR change the field direction, the changes of the different components is completely compensate each other, and the signal remains unchanged). In the PBR the wing linear polarization for a given multiplet component depends on the magnetic field and its direction, although the wings are not influenced by the Hanle effect.

However, far from the multiplet the polarization approaches the zero-field value in both the PBR and ZR. This can be easily understood from the principle of spectroscopic stability. Far from the system of lines we can neglect all quantum numbers that govern the inner structure of the system. Thus we can consider the system as a single line with fixed initial and final states, for which there is neither the PBE nor the Hanle effect. For example, far from the multiplet resonances the spin can be set to zero, so that the multiplet will appear as a singlet. Thus, in the wings of the multiplet we can neglect both the PBE and the Hanle effect, while within the line system all changes due to the PBE are still important.

Note that only full inclusion of all rotational branches while taking into account all possible interferences allows us to obtain the same signal in the wings of a doublet for the PBR and ZR. This equality of the wing polarization can be used to test the computational algorithms.

\subsection{Line core depolarization}

Calculations for the entire multiplet reveal that even in the zero field case (thus without Hanle and PBE) there is depolarization in the line core as compared with the wing polarization. This is usually not the case in calculations with one multiplet component. Indeed, in zero field all coefficients of the phase matrix for scattering in a single line have the same profiles and the linear polarization degree $Q / I$ does not depend on frequency.

The reason for the core depolarization is the interferences between the scattering processes via different fine structure $J$-levels of the upper state (the third sum in Eq. (10)), because we can neglect them in the doublet core but have to take them into account in the doublet wings. To understand this we firstly note that the distance between the fine structure levels is generally much bigger than their natural widths (Fig. 1). Let us consider one photon scattered in the doublet core (within several Doppler widths) and choose the reference system for a molecule at which it was scattered. There are eight different processes, corresponding to rotational branches (see above) that can contribute to this scattering. Since the Doppler width is much larger than the natural width, there is a large probability that the scattering occurs close to one of these processes (within several natural widths) but with a different wavelength mainly due to the Doppler broadening. As the distance between lines of different branches is much larger than the natural width, we can neglect all contributions from other scattering processes. It implies that we can neglect the interferences, despite the fact that all components are so close in the spectrum, that we cannot resolve them. This is because the interferences have to be taken into account before convolving with the Doppler profile, as only the natural width is important.

Consider now the photon scattered far from the multiplet resonances. Since the Doppler profile is decreasing much faster then the Lorentz profile, there is a big probability that this photon was scattered far from the multiplet because of the uncertainty in the energy level, rather than because of the Doppler broadening. Thus, even in the molecular reference system, the photon was scattered far from the doublet. In this case, however, we cannot isolate the main component of the scattering process as we did before but we need to take into account the contributions from all components, which introduces the interferences terms.

To conclude, the depolarization in the multiplet core is due to the gradual disappearance of the interference between different fine structure transitions. It is quite analogous to the Hanle effect, when decreasing interferences between different magnetic subcomponents in a magnetic field causes the depolarization in the line core.

\subsection{Doublet line profiles in the PBR}

Here we present calculated scattering profiles of $\mathrm{CN}$ and $\mathrm{MgH}$ transitions for different magnetic fields and Doppler widths. For calculating the lifetimes we use oscillator strengths 

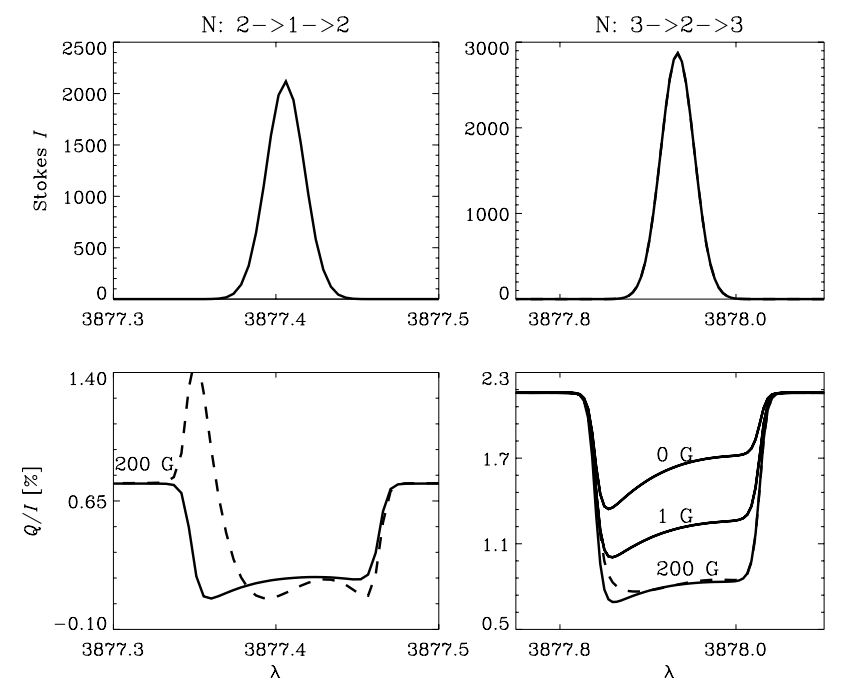

Fig. 3. Scattering profiles for a whole doublet, in the $(0,0)$ band of the $\mathrm{CN}$ violet system. Solid and dashed curves represent the PBR and ZR cases respectively. Left panels: $P(2), N=2 \rightarrow 1 \rightarrow 2$, for $200 \mathrm{G}$ field. Right panels: $P(3), N=3 \rightarrow 2 \rightarrow 3$, for 0,1 and $200 \mathrm{G}$ fields. Note that the PBE and ZE curves for $0 \mathrm{G}$ and $1 \mathrm{G}$ superimpose, so they are undistinguishable, while the curves for $200 \mathrm{G}$ can be easily seen separately. The magnetic field is perpendicular to the line of sight and is in the plane of the solar surface. The Doppler width $\Delta \lambda_{\mathrm{D}}=25 \mathrm{~m} \AA$, the Voigt parameter $a=0.0001$. Unpolarized incident radiation field and single scattering at angle $90^{\circ}$ are assumed. Collisional depolarization and continuum opacity are neglected.

from Knowles et al. (1988); Wallace et al. (1999); Kirby et al. (1979). In all plots we assume an unpolarized incident radiation field and single scattering at an angle of $90^{\circ}$. Collisional depolarization and continuum opacity are neglected. As in all plots we choose the magnetic field direction to be perpendicular to the line of sight, and the Stokes $U$ and $V$ signals equal to zero. The profiles are calculated for a realistic Doppler width $\lambda_{\mathrm{D}}=25 \mathrm{~m} \AA$ which is much larger than the distance between the fine structure components, or assuming a very narrow width $\lambda_{\mathrm{D}}=2.5 \mathrm{m \AA}$ to illustrate the profile asymmetries. Note that in all figures we present the Stokes $I$ profiles only for the $200 \mathrm{G}$ magnetic field. As we here only deal with ninety degree scattering, the Stokes I profiles slightly change due to the Hanle effect. These Hanle modifications almost completely vanish when taking into account all scattering angles and modest anisotropy of incident radiation field for the real Sun.

In Fig. 3 we present doublet scattering profiles for $P(2)(N=$ $2 \rightarrow 1 \rightarrow 2)$ and $P(3)(N=3 \rightarrow 2 \rightarrow 3)$ transitions in the presence of a horizontal magnetic field. We assume the spinrotational constant for the upper state $\gamma_{\mathrm{SR}}=0.0171 \mathrm{~cm}^{-1}$ and for lower state $\gamma_{\mathrm{SR}}=0.0073 \mathrm{~cm}^{-1}$. The PBE profiles differ significantly from the ZR case. First of all, the PBE modifies the profile shape. Furthermore, the integrated line polarization differs in general in the PBR and ZR cases. In the case of the $P(3)$ transition the main manifestation of the Hanle effect, i.e. depolarization in the line core, is easily visible in both the ZR calculations (dashed) and the full PBE (solid) case. For a magnetic field of $1 \mathrm{G}$ the PBE is too weak (cf. Figs. 1 and 2) and there is no difference between the ZR and PBR profiles. For a magnetic field of $200 \mathrm{G}$ the PBR and ZR polarization differ slightly across the whole line profile. The biggest difference is near the line wings. However as the absolute signal there is already quite small (see corresponding Stokes I profile), such a difference is not very important and will hardly be observable because of the continuum
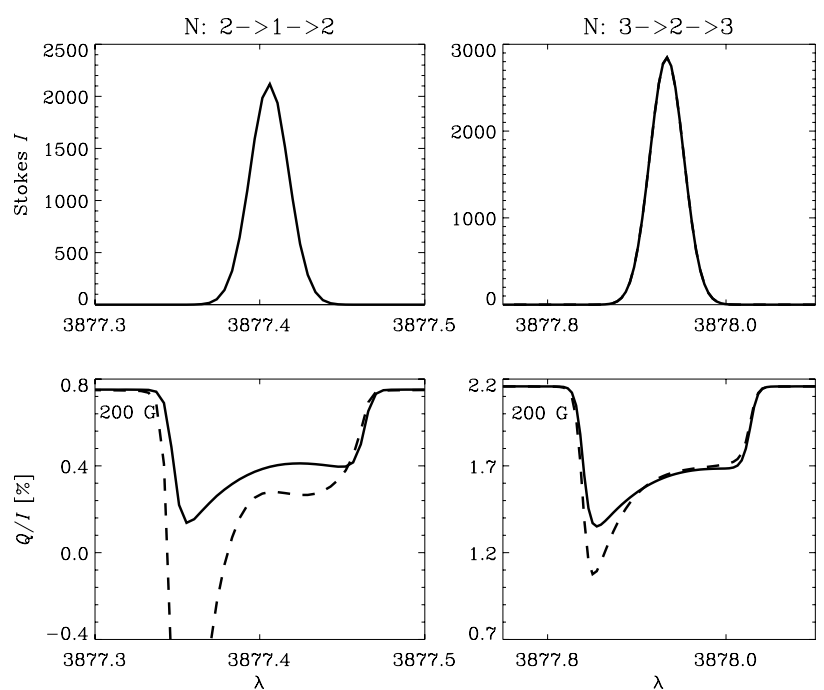

Fig. 4. Same as Fig. 3 but for a magnetic field direction perpendicular to the solar surface and the line of sight. There is no Hanle effect in the usual sense in this case. In both panels only the curves for $200 \mathrm{G}$ are shown.

polarization. In the case of the $P(2)$ transition the differences between the PBR and ZR are much bigger. The core polarization in the PBR is bigger than in the ZR. Near the line wings the relative contributions from the satellite and forbidden branches (which are induced by the PBE) become important. As a result the ZR and PBR polarization levels are completely different. Note that the $P(2)$ transition is somewhat special: in the $\mathrm{ZR}$ the effective scattering polarizability $W_{2}$ of the $P_{2}(2)$ component is zero and this component does not contribute to the polarization. In the PBR the situation is changed, and there is quite significant redistribution of polarization between the doublet components.

In Fig. 4 we present scattering profiles for the same transitions as in Fig. 3, but for a vertical magnetic field. It is wellknown that the Hanle effect is absent for a vertical field direction (thus in contrast to the previous case, the polarization for the magnetic fields of 0 and $1 \mathrm{G}$ will be the same). In the case of $P(2)$ the $\mathrm{ZR}$ profiles for $200 \mathrm{G}$ are quite close to the $0 \mathrm{G}$ profile in the line core, but they differ significantly near the blue wing. The PBR polarization for $200 \mathrm{G}$ is significantly different from both the zero field polarization and from the $200 \mathrm{G} \mathrm{ZR}$ polarization over the whole profile. Like in the horizontal field case the PBR increases the line core polarization. For the $P(3)$ transition the changes are smaller and noticeable only near the line wings.

In Fig. 5 scattering profiles for the $R(1)$ transition are presented for two values of the Doppler width in presence of a horizontal field. For a Doppler width of $2.5 \mathrm{~m} \AA$ (right panel) it is possible to distinguish different components of the fine structure (we mark them in the plot with their zero field wavelengths). We can see that the difference between the ZR and PBR is mainly due to transition amplitudes changes for the PBE. If we increase the Doppler width up to $25 \mathrm{~m} \AA$ all these differences vanish almost completely. As shown in the left panel of Fig. 5 only very small changes of the polarization near the line wings may be noticed.

For larger $J$ values the energy difference between the fine structure levels increases, so that the efficiency of the PBE decreases. Therefore the distinction between the PBR and ZR becomes smaller with increasing $J$-value. We also find that for small $N$ numbers the PBE more strongly influences the P-branch than the R-branch transitions. This is due to the larger effective 

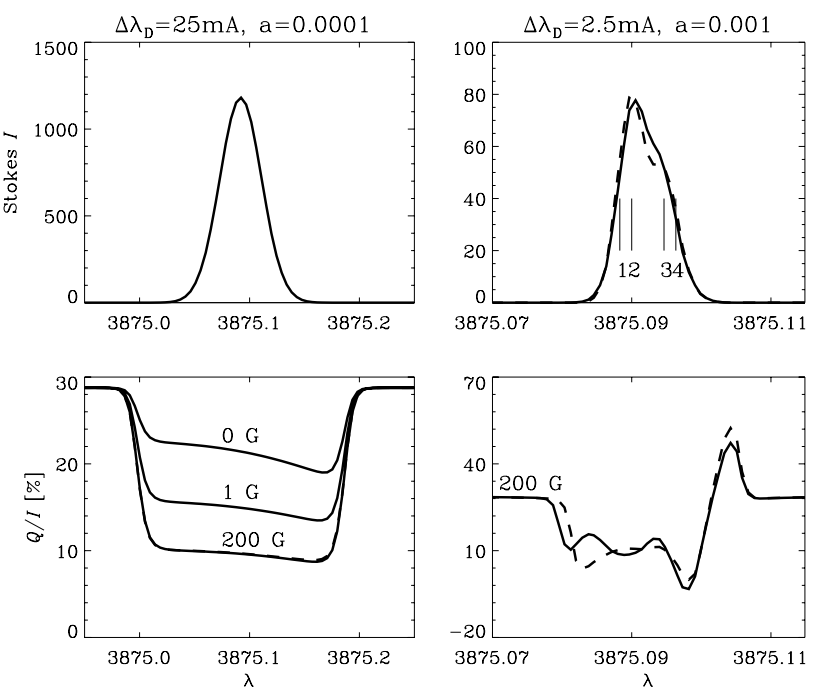

Fig. 5. Scattering profiles for the $\mathrm{R}(1), N=1 \rightarrow 2 \rightarrow 1$ transition. Left panels: Realistic Doppler width $\Delta \lambda_{\mathrm{D}}=25 \mathrm{~m} \AA$ and $a=0.0001$, curves for $0,1,200 \mathrm{G}$ fields. Note that like in Fig. 3 the PBE and ZE curves for $0 \mathrm{G}$ and $1 \mathrm{G}$ superimpose, while the curves for $200 \mathrm{G}$ might be seen separately (but the difference between them is much smaller than in Fig. 3). Right panels: very small Doppler width $\Delta \lambda_{\mathrm{D}}=2.5 \mathrm{~m} \AA$ and $a=0.001$, curves only for a field of $200 \mathrm{G}$. In the top and right panel the different components of the doublet are marked: " 1 " for ${ }^{R} S_{12}(0.5)$, "2" for $R_{1}(1.5)$, "3" for $R_{2}(0.5)$, "4" for ${ }^{R} Q_{21}(1.5)$.
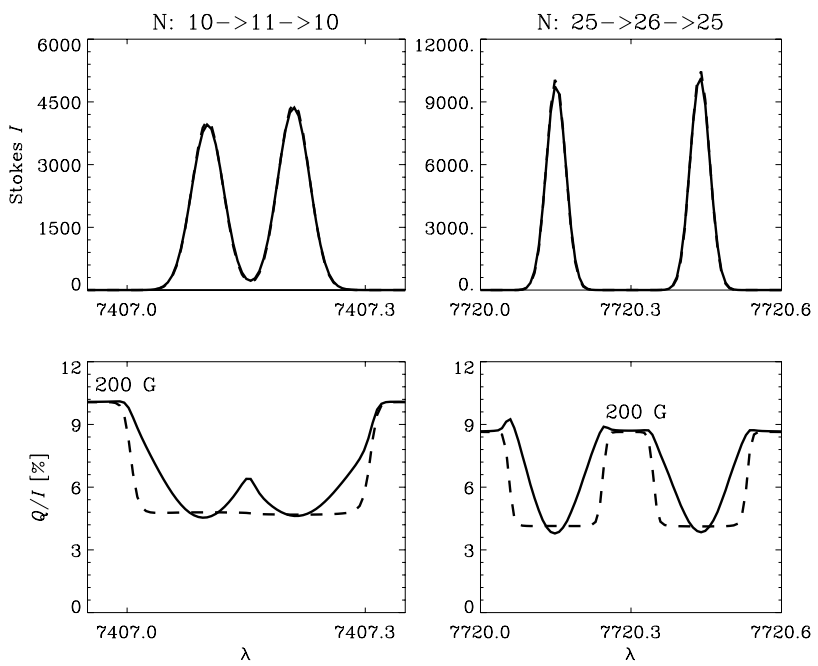

Fig. 6. Scattering profiles for transitions in the $(0,7)$ band of the $\mathrm{MgH}$ $B^{\prime 2} \Sigma-X^{2} \Sigma$ system, assuming a horizontal field. Left panel: $R(10)$, $N=10 \rightarrow 11 \rightarrow 10$ transition. Right panel: $R(25), N=25 \rightarrow 26 \rightarrow 25$ transition. Solid and dashed lines represent the PBR and ZR calculations, respectively. Doppler width $\Delta \lambda_{\mathrm{D}}=25 \mathrm{~m} \AA$ field strength $200 \mathrm{G}$, and spin-rotational constant $\gamma_{\mathrm{SR}}=10^{-4} \mathrm{~cm}^{-1}$.

scattering polarizability of the satellite branches ${ }^{P} Q_{12}$ and ${ }^{P} O_{21}$, which despite their relative weakness contribute significantly to the polarization of $P$-branch transitions.

We can expect a bigger change due to the PBE if the separation between the interacting levels is smaller. The $\mathrm{MgH}$ $B^{\prime 2} \Sigma-X^{2} \Sigma$ system is an interesting example. The multiplet splitting in the upper state of this transition has not been resolved (cf. Balfour \& Cartwright 1976), implying that the spinrotational constant $\gamma_{\mathrm{SR}}$ should be quite small. In Fig. 6 we show an example of calculations assuming $\gamma_{\mathrm{SR}}=10^{-4} \mathrm{~cm}^{-1}$. The PBR calculations significantly differ from the $\mathrm{ZR}$, even for quite big $N$-numbers, when the doublet structure is well seen (mainly due to the fine structure splitting of the lower $X^{2} \Sigma$ state with $\left.\gamma_{\mathrm{SR}}=0.019 \mathrm{~cm}^{-1}\right)$.

The PBR-profiles of the fine structure components of $\mathrm{MgH}$ in Fig. 6 are much narrower than the ZR-profiles. It has to do with the separation of the magnetic sublevels in the PBE by spin projection (see Sect. 2). Note also that in the PBR-calculations different magnetic sublevels cannot intersect each other. In the ZR-calculations such intersections, implying a strong increase of the interference terms at certain magnetic field strengths, are possible. As a result, in the ZR the shapes of the $Q / I$ signal and the integrated depolarization do not change monotonously with the field, as it does in the PBR.

\section{Net depolarization in the PBR}

For zero magnetic field all contributions to the Jones matrix from scattering processes via different magnetic substates of one $J$ state have the same phase (cf. Eq. (5)). This implies maximum contributions the interference terms (second sum in Eq. (10)). In a magnetic field, because of the level splitting, the interference terms in the line core become smaller and asymptotically disappear when the magnetic splitting becomes significantly larger than the level's natural width. The Hanle effect in such fields is called saturated. Note that in the line wings there is no decrease of the interference terms in a magnetic field, and the situation is fully identical to the zero-field case, as we saw before from the principle of spectroscopic stability.

Depolarization due to the saturated Hanle effect does not depend on the magnetic field in the ZR calculations. In the present section we show that in the PBR the situation is different.

\subsection{Hanle depolarization of one multiplet component}

In the weak-field approximation $\left(\Delta \lambda_{\mathrm{Z}} \ll \Delta \lambda_{\mathrm{D}}\right)$ we can separate the angular and wavelength dependent parts of the phase matrix. For a given angular distribution of the magnetic field vector with the strength $B$ the depolarization factor due to the Hanle effect (Stenflo 1982)

$W_{\mathrm{H}}(\lambda, B)=\frac{Q / I(\lambda, B)}{Q / I(\lambda, 0)}$

which depends on the field strength $B$ and wavelength $\lambda$. It equals to unity in the line wings and is usually smaller than one in the line core. Also in the PBR $W_{\mathrm{H}}(\lambda, B)$ retains its meaning.

If the fine structure splitting is either much smaller or much larger than the Doppler width it is possible to neglect the dependence on wavelength in the line core and characterize the depolarization for a fixed magnetic field just by $W_{\mathrm{H}}(B)$. Such a quantity was introduced by (Stenflo 1982) and is widely used in different applications (cf. Faurobert \& Arnaud 2003; Berdyugina $\&$ Fluri 2004) to explore turbulent fields. This case is covered by the well-known expression for $W_{\mathrm{H}}(B)$ given by Stenflo (1982) and Landi Degl'Innocenti \& Landi Degl'Innocenti (1988).

In the weak field regime $W_{\mathrm{Z}}(B)$ also governs the integrated depolarization, since most of the radiation comes from the line core. Since the PBE modifies the shape of $W_{\mathrm{H}}(\lambda, B)$, we calculate the integrated depolarization in the PBE as follows:

$\bar{W}_{\mathrm{H}}(B)=\int_{\Delta \lambda} Q(\lambda, B) \mathrm{d} \lambda / \int_{\Delta \lambda} Q(\lambda, 0) \mathrm{d} \lambda$, 
Table 1. Depolarization factors and integrated polarization for the $P_{1}(1.5)$ and $R_{2}(0.5)$ lines of the $\mathrm{CN}(0,0)$ band. The $W_{\mathrm{H}}$ values have been calculated with Eq. (2.12) from Stenflo (1982).

\begin{tabular}{c|cc|cc}
\hline \hline & \multicolumn{2}{|c|}{$P_{1}(1.5)$} & \multicolumn{2}{c}{$R_{2}(0.5)$} \\
$H,[\mathrm{G}]$ & $W_{\mathrm{H}}$ & $\bar{W}_{\mathrm{H}}(B)$ & $W_{\mathrm{H}}$ & $\bar{W}_{\mathrm{H}}(B)$ \\
\hline 0 & 1.000 & 1.000 & 1.000 & 1.000 \\
1 & 0.376 & 0.375 & 0.689 & 0.687 \\
5 & 0.209 & 0.209 & 0.260 & 0.259 \\
10 & 0.202 & 0.202 & 0.216 & 0.215 \\
20 & 0.200 & 0.201 & 0.204 & 0.202 \\
40 & 0.200 & 0.204 & 0.201 & 0.195 \\
60 & 0.200 & 0.210 & 0.200 & 0.188 \\
80 & 0.200 & 0.222 & 0.200 & 0.182 \\
100 & 0.200 & 0.240 & 0.200 & 0.175 \\
120 & 0.200 & 0.265 & 0.200 & 0.170 \\
140 & 0.200 & 0.295 & 0.200 & 0.166 \\
160 & 0.200 & 0.328 & 0.200 & 0.163 \\
180 & 0.200 & 0.363 & 0.200 & 0.162 \\
200 & 0.200 & 0.396 & 0.200 & 0.161 \\
\hline
\end{tabular}

where the range of the integration covers several Doppler widths. Note that this quantity does not use the weak field approximation (although in the weak field approximation it is similar to $W_{\mathrm{H}}(B)$ ).

In Table 1 we present $\bar{W}_{\mathrm{H}}(B)$ in the PBR for $R_{2}(0.5)$ and $P_{1}(1.5)$ lines of the $\mathrm{CN}(0,0)$ band, assuming a turbulent field, and compare them with $W_{\mathrm{H}}(B)$. The saturated Hanle effect for these lines appears already at a field strength of $B_{\text {sat }} \approx 10 \mathrm{G}$. We can see that the PBE introduces additional depolarization in the $R$ and polarization in the $P$ branch lines. This is because the transition amplitudes still depend on the magnetic field strength even in the saturated Hanle regime. Therefore the function $\bar{W}_{\mathrm{H}}(B)$ does not reach an asymptotic value but continues to vary with magnetic field strength.

\subsection{Hanle depolarization of a whole multiplet}

The depolarization in the line core of a doublet is due to two types of interferences between different magnetic components and between different components of the fine structure (see Sect. 4.2). Therefore one can write:

$W_{\text {tot }}(\lambda, B)=W_{\mathrm{F}}(\lambda, B) W_{\mathrm{H}}(\lambda, B)$

where $W_{\mathrm{F}}(\lambda)$ is governs the fine structure depolarization and $W_{\mathrm{H}}(\lambda, B)$ (from Eq. (11)) governs the Hanle depolarization. Note that because of PBE $W_{\mathrm{F}}$ also depends on the magnetic field strength.

To study the influence of PBE on the depolarization we introduce the integrated quantity:

$\bar{W}_{\text {tot }}(B)=\frac{\int Q(\lambda) \mathrm{d} \lambda / \int I(\lambda) \mathrm{d} \lambda}{(Q / I)_{\text {wings }}}$.

We can again rewrite this as a product of two depolarization factors:

$\bar{W}_{\text {tot }}(B)=\bar{W}_{\mathrm{F}}(B) \bar{W}_{\mathrm{H}}(B)$.

Because of the interference between different fine structure components $\bar{W}_{\text {tot }}(B)$ differs from unity even in the zero field case. In the present section we will use the both $\bar{W}_{\text {tot }}(B)$ and $\bar{W}_{\mathrm{H}}(B)$ values.
The efficiency of the Hanle effect strongly depends on the Landé factors of the upper state, which determine the magnetic displacements of the sublevels and hence the phase shifts. For molecular lines (and in particular for $\mathrm{CN}$ ) the Landé factor usually decreases as the $J$ number increases (Berdyugina \& Solanki 2002). Therefore transitions with small $J$ are particularly strongly influenced by a magnetic field and quickly reach the saturated Hanle regime. For all $\mathrm{CN}$ transitions discussed above the saturated Hanle effect occurs already at field strengths of 10-20 G. Thus for such fields the interference between different magnetic sublevels in the line core is lost, and as we can see from Fig. 1 they will never come back again. For lines with larger $J$ values the saturated Hanle effect requires in stronger magnetic fields but, due to the increase of the fine structure splitting with $J$, the influence of the PBE for such lines is smaller.

Thus for transitions with small $J$ numbers we have the following situation. In the line core in case of zero field there is interference between different magnetic sublevels of each fine structure component, but there is no interference between the different fine structure components. In a field of 10-20 G the saturated Hanle effect occurs, i.e. there is no interference in the line core at all, but in the line wings both types of interference are present. For such fields the PBE is very small and we can completely neglect it. Therefore when the PBE becomes important we already have reached the saturated Hanle effect, so that we can calculate the depolarized signal while considering all scattering processes independently from each other without any interferences, i.e. neglecting the second and third sums in Eq. (10).

We can now classify the changes in the Stokes $Q$ profiles caused by the PBE into two types. Most important is the change of $\bar{W}_{\text {tot }}(B)$, which governs the total line depolarization. Such changes create well-defined observational signatures. They can occur only if the transition strengths change due to magnetic field. This is possible only because of the PBE.

The second type of change occurs because the PBE introduces other displacements, and at the given frequency the contributions from the various scattering processes are different in the PBR and in the ZR, the polarization is redistributed over the whole profile. Therefore the shape of the profile is changed, while the total depolarization remains the same. Obviously it is much more difficult to identify this type of change from observational data.

\subsection{Saturated polarization in vertical and horizontal fields}

The calculation of the saturated, integrated Stokes parameters is much easier than dealing with the general case. It is even possible to give relatively simple analytical expressions for vertical and horizontal fields. The ratio will always have the form

$\frac{\int Q_{\mathrm{sat}}(\lambda) \mathrm{d} \lambda}{\int I_{\mathrm{sat}}(\lambda) \mathrm{d} \lambda}=\frac{A-B}{A+B}$,

where for a magnetic field perpendicular to the solar surface (as in Fig. 4)

$A_{\text {vert }}=\sum_{M, J_{\mathrm{i}}, J_{\mathrm{u}}, J_{\mathrm{f}}} f_{M, M \pm 1}\left(\frac{f_{M \pm 1, M}+f_{M \pm 1, M \pm 2}}{2}\right)$
$B_{\mathrm{vert}}=\sum_{M, J_{\mathrm{i}}, J_{\mathrm{u}}, J_{\mathrm{f}}} f_{M, M \pm 1} f_{M \pm 1, M \pm 1}$. 
For the case when the magnetic field is perpendicular to the line of sight and lies in the plane of solar surface (Figs. 3 and 5)

$$
\begin{aligned}
A_{\text {hor }}= & \sum_{M, J_{\mathrm{i}}, J_{\mathrm{u}}, J_{\mathrm{f}}} f_{M, M} f_{M, M}+\frac{f_{M, M \pm 1} f_{M \pm 1, M \pm 1}}{2} \\
B_{\text {hor }}= & \sum_{M, J_{\mathrm{i}}, J_{\mathrm{u}}, J_{\mathrm{f}}} f_{M, M}\left(\frac{f_{M, M+1}+f_{M, M-1}}{2}\right) \\
& +f_{M, M \pm 1}\left(\frac{f_{M \pm 1, M}+f_{M \pm 1, M \pm 2}}{4}\right) .
\end{aligned}
$$

Here, in all bilinear products of oscillator strengths the first factor refers to absorption ( $\mathrm{i} \rightarrow \mathrm{u}$ transition), the second to emission $(\mathrm{u} \rightarrow \mathrm{f}$ transition). Let us emphasize that these expressions are valid only in the saturated Hanle regime. They fail for weaker fields (where we have to account for the all interferences).

\subsection{Single profile approximation}

We can make one more simplification, namely assume that the Doppler width is much larger than the distance between the fine structure components. In this case we can consider all contribution to the Jones matrix (see Eqs. (4) and (5)) as having the same frequency profile. Thus all elements of the coherency matrix in Eq. (6) will also have the same profile. The profile function will therefore divide out when forming the fractional polarization, so that the ratio $Q / I$ will be a constant.

As we can see from Figs. $3-5$ for the $\lambda_{D}=25 \mathrm{~m} \AA$ case this approximation is quite good (for curves corresponding to a magnetic field of $200 \mathrm{G}$ there is a flat, almost horizontal part of the profile near the line core).

With both our approximation (the omission of the interferences and the single profile approximation) Stokes $Q$ in the line core is proportional to Stokes $I$. Because of this we do not need the integration in Eq. (14) but can write the saturated $\bar{W}_{\text {tot }}(B)$ as

$\bar{W}_{\text {tot }}^{\text {sat }}(B)=\frac{\left[Q\left(\lambda_{\text {core }}\right) / I\left(\lambda_{\text {core }}\right)\right]_{\text {sat }}}{(Q / I)_{\text {wings }}}$.

Thus in case of the saturated Hanle effect and large Doppler broadening, $\bar{W}_{\text {tot }}^{\text {sat }}(B)$ gives us the ratio between the core and wing polarizations.

\subsection{Example calculations for $\mathrm{CN}$ and $\mathrm{MgH}$}

In Figs. 7-9 we present calculations of $\bar{W}_{\text {tot }}^{\text {sat }}(B)$ for the $\mathrm{CN}$ transitions, using Eqs. (16)-(18) to calculate the numerator in the ratio of Eq. (14). Note that all curves in these figures are valid only for fields stronger than about $10 \mathrm{G}$, since for weaker fields the Hanle effect is not saturated.

In Figs. 7 and 8 the dependence of $\bar{W}_{\text {tot }}^{\text {sat }}(B)$ on the magnetic field strength is shown. The depolarization changes with magnetic field in the PBR, while in the ZR it remains constant (because in the ZR only the position of the lines but not their strengths change).

In a vertical field (Fig. 7) the depolarization for all transitions asymptotically approaches unity with increasing field strength. The reason is that for a vertical field only interferences between different components of the doublet are important. For example, for zero field we only have to take into account interferences between magnetic sublevels of states with different $J$ numbers. As these sublevels belong to different $J$ states, they will get opposite spin projections on the magnetic field direction $\mu_{\mathrm{S}}$ when the field

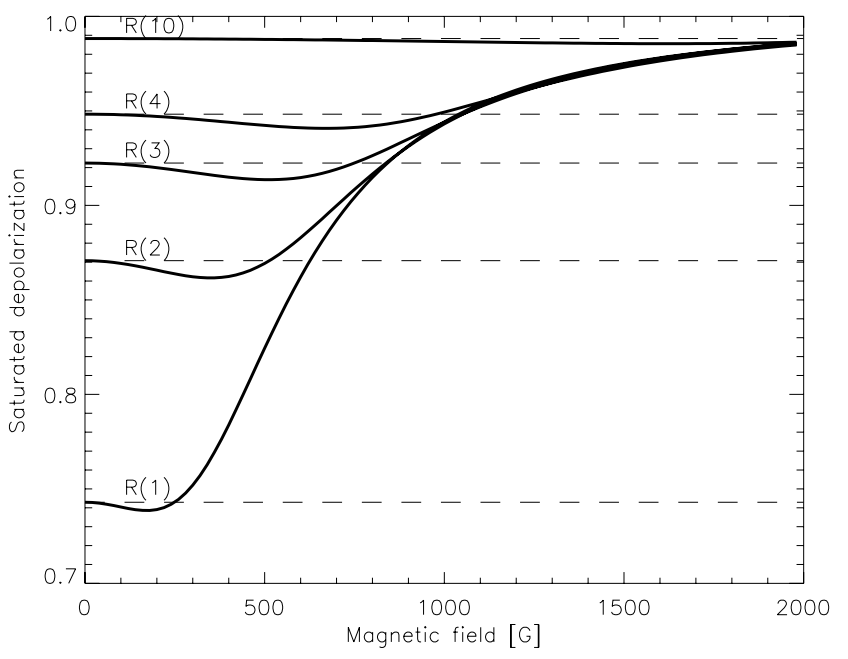

Fig. 7. The dependence of saturated depolarization on magnetic field strength. The $N$ numbers of the transitions are indicated in the plot. The magnetic field is perpendicular to the solar surface. The solid curves represent the PBR case, the dashed lines the ZR case.

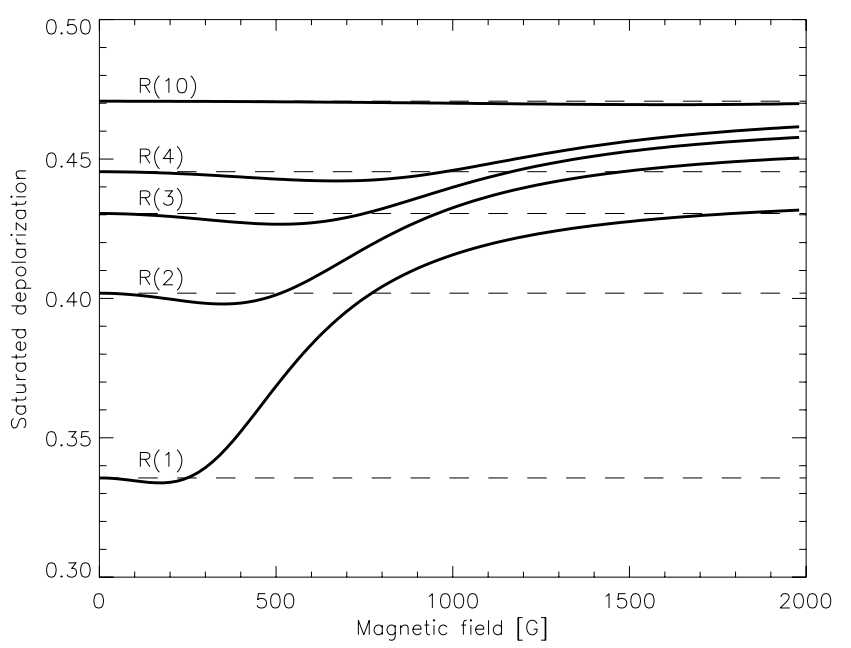

Fig. 8. The same as Fig. 7, but for a magnetic field vector perpendicular to the line of sight and in the plane of the solar surface.

becomes strong (cf. Fig. 1). The strengths of such transitions will decrease with field strengths and asymptotically becomes forbidden in the total PBE (see bottom right panel of Fig. 2). Thus, the interference between the different components of a doublet disappears (now, in contrast to the previous discussion, over the whole profile, including line wings).

For a horizontal field (Fig. 8) the interference between the different components of the doublet also goes to zero for a strong magnetic fields (this property does not depend on field direction), but the interference between the different magnetic sublevels of the same doublet component remains significant. Therefore the asymptotic values of depolarization are different for the different lines and do not approach unity as for the vertical field.

The dependence of the saturated depolarization $\bar{W}_{\text {tot }}^{\text {sat }}(B)$ on field direction is presented in Fig. 9. One can see that the changes due to the PBE are larger for the above considered above cases - vertical and horizontal magnetic fields.

Significant modifications of the depolarization due to the PBE occurs only in relatively strong magnetic fields $\geq 500 \mathrm{G}$. 

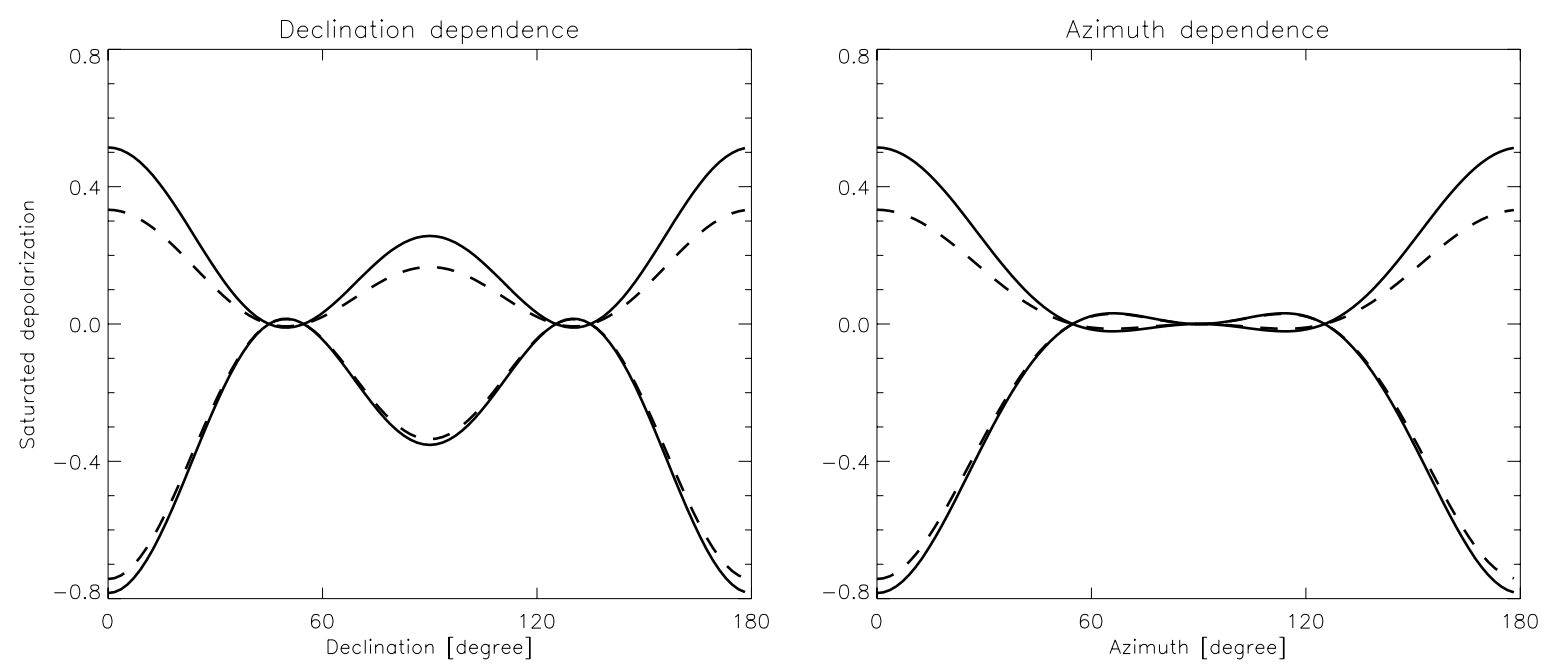

Fig. 9. Dependence of saturated depolarization on field direction. Left panel: dependence on declination (angle with the plane which is perpendicular to the solar surface), when the azimuth (angle with the plane that is perpendicular to the direction on the observer and contains the normal to the solar surface) equals zero. Right panel: dependence on azimuth (when the declination equals zero). Curves for a $P(2), N=2 \rightarrow 1 \rightarrow 2$ transition in a $200 \mathrm{G}$ field and for a $R(1), N=1 \rightarrow 2 \rightarrow 1$ in a $400 \mathrm{G}$ field. We have changed the sign of the depolarization for the $1 \rightarrow 2 \rightarrow 1$ curves to make plots more clear. The solid curves represent the PBR case, the dashed curves the ZR case.

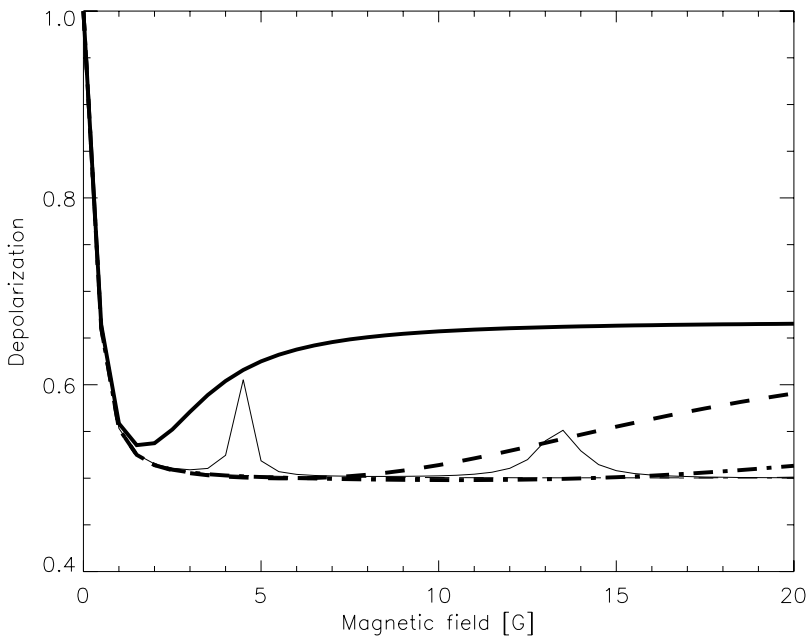

Fig. 10. Dependence of depolarization on the magnetic field strength for the $(0,7)$ band of the $\mathrm{MgH} B^{\prime 2} \Sigma-X^{2} \Sigma$ system for different values of the spin-rotational constant $\gamma_{\mathrm{SR}}$, for the transitions $R(1), N=1 \rightarrow$ $2 \rightarrow 1$. Continuous lines represent $\gamma_{\mathrm{SR}}=10^{-4} \mathrm{~cm}^{-1}$, dashed lines $\gamma_{\mathrm{SR}}=$ $5 \times 10^{-4} \mathrm{~cm}^{-1}$, dashed-dotted lines for $\gamma_{\mathrm{SR}}=10^{-3} \mathrm{~cm}^{-1}$. Thick curves represent the PBR case, thin the ZR case.

Only for $N=2 \rightarrow 1 \rightarrow 2$ there is a quite strong effect already for fields of 100-200 G. An absolutely different situation occurs for the $\mathrm{MgH} B^{\prime 2} \Sigma-X^{2} \Sigma$ system. Since the spinrotational constant of the $B^{\prime 2} \Sigma$ state might be quite small (see Sect. 4), the PBE becomes important before Hanle saturation. In this case we cannot neglect the interferences, and the method described in Sect. 5.2 does not work. Therefore it is necessary to calculate the full integrals of Eq. (12), which is quite time consuming. In Fig. 10 we show the dependence of the depolarization $\bar{W}_{\mathrm{H}}(B)$ defined by Eq. (12) on the field strength for several possible $\gamma_{\mathrm{SR}}$ values, assuming the $N=2 \rightarrow 1 \rightarrow 2$ transition. It is obvious that for smaller $\gamma_{S R}$ the PBE occurs at weaker fields, and for $\gamma_{S R}=10^{-4} \mathrm{~cm}^{-1}$ it happens even before the Hanle effect is saturated. For bigger $N$ values only the scale of the magnetic field axis is changed, since the Landé factors decrease linearly with $J$, while the spin-rotational splitting is proportional to $J$. Non monotonous shapes of the ZR curves appear due to level crossings (which lead to increasing interference), while for the PBR level-crossing is impossible and all curves have monotonous shapes.

\section{Conclusions}

We have presented here for the first time the theory of the molecular Hanle effect in the PBR and illustrated it with examples for the CN $B^{2} \Sigma-X^{2} \Sigma$ and $\mathrm{MgH} B^{\prime 2} \Sigma-X^{2} \Sigma$ systems. Based on our results we can draw the following conclusions:

- Since Landé factors of magnetic sublevels and transition amplitudes are strongly modified by the PBE, the phases and amplitudes of the Jones matrix elements will be changed.

- The PBE influences the scattering polarization over the whole system of perturbed transitions. However, far from the multiplet resonances, the polarization approaches the zerofield value in agreement with the principle of spectroscopic stability.

- Our calculations for the $\mathrm{CN}$ violet system, widely observable in the second solar spectrum, show that the line profiles of the individual doublet components significantly change due to the PBE already for a magnetic field strength of $100 \mathrm{G}$ for levels with small $J$ values. These changes are however smeared by Doppler broadening and influence from the second doublet component.

- Detailed calculations of the CN violet system doublets for different field directions show that the PBE is more important for $\mathrm{P}$ branch transitions than for the $\mathrm{R}$ branch.

- The upper state multiplet splitting of the $\mathrm{MgH} B^{\prime 2} \Sigma-X^{2} \Sigma$ system is still unresolved, implying a small splitting and thus a strong PBE. Assuming a spin-rotational constant $\gamma_{\mathrm{SR}}=$ $10^{-4} \mathrm{~cm}^{-1}$ we find a considerable difference between the PBR and ZR even for levels with relatively large $J$ values

- The depolarization of the $\mathrm{MgH}$ transitions due to the Hanle effect is considerably influenced by the PBE already at field strengths of $10 \mathrm{G}$. 
- We have introduced a simple method for calculations in the saturated Hanle regime (neglecting the interferences between different magnetic and fine structure transitions) and have shown that in the PBR the saturated polarization is magnetic field dependent, in contrast to the ZR. We discuss its dependence on magnetic field strength and direction.

Acknowledgements. This work has been supported by SNF grant 20002103696. S.B. acknowledges the EURYI Award, from the ESF and SNF, grant PE002-104552.

\section{References}

Asensio Ramos, A., \& Trujillo Bueno, J. 2005, ApJ, 635, L109

Asensio Ramos, A., \& Trujillo Bueno, J. 2006, ApJ, 636, 548

Balfour, W. J., \& Cartwright, H. M. 1976, A\&AS, 26, 389

Berdyugina, S. V., \& Solanki, S. K. 2002, A\&A, 385, 701

Berdyugina, S. V., \& Fluri, D. M. 2004, A\&A, 417, 775

Berdyugina, S. V., Stenflo, J. O., \& Gandorfer, A. 2002, A\&A, 388, 1062

Berdyugina, S. V., Braun, P. A., Fluri, D. M., \& Solanki, S. K. 2005, A\&A, 444, 947

Bommier, V. 1980, A\&A, 87, 109

Bommier, V., Landi Degl'Innocenti, E., \& Molodij, G. 2006, in Solar Polarization, Proc. 4rd SPW, ed. R. Casini, \& B. W. Lites, ASP Conf. Ser., in press
Faurobert, M., \& Arnaud, J. 2003, A\&A, 412, 555

Herzberg, G. 1950, Molecular spectra and molecular structure, Vol. 1, Spectra of diatomic molecules (New York: Van Nostrand Reinhold) 2nd edn.

Hill, E. L. 1929, Phys. Rev., 34, 1507

Kirby, K., Saxon, R. P., \& Liu, B. 1979, ApJ, 231, 637

Knowles, P. J., Werner, H.-J., Hay, P. J., \& Cartwright, D. C. 1988, J. Chem. Phys., 89, 7334

Kovács, I. 1969, Rotational Structure in the Spectra of Diatomic Molecules (London: Adam Hilger Ltd.)

Landi Degl'Innocenti, E. 2003, in Solar Polarization, Proc. 3rd SPW, ed. J. Trujillo Bueno, \& J. Sánchez Almeida, ASP Conf. Ser., 307, 164

Landi Degl'Innocenti, M., \& Landi Degl'Innocenti, E. 1988, A\&A, 192, 374

Landi Degl'Innocenti, E. L., \& Landolfi, M. 2004, Physics of Solids and Liquids

Landolfi, M., \& Landi Degl'Innocenti, E. 1985, Sol. Phys., 98, 53

Schadee, A. 1978, JQRST, 19, 517

Shapiro, A. I., Fluri, D. M., Berdyugina, S. V., \& Stenflo, J. O. 2006, in Solar Polarization, Proc. 4rd SPW, ed. R. Casini, \& B. W. Lites, ASP Conf. Ser., in press

Stenflo, J. O. 1982, Sol. Phys., 80, 209

Stenflo, J. O. 1994, Solar Magnetic Fields (Dordrecht: Kluwer)

Stenflo, J. O. 1997, A\&A, 324, 344

Stenflo, J. O. 1998, A\&A, 338, 301

Trujillo Bueno, J. 2003, in Solar Polarization, Proc. 3rd SPW, ed. J. Trujillo Bueno, \& J. Sánchez Almeida, ASP Conf. Ser., 307, 407

van Vleck, J. H. 1926, Phys. Rev., 28, 980

Wallace, L., Hinkle, K., Li, G., \& Bernath, P. 1999, ApJ, 524, 454 